family. Thus, by Theorem 3.1, every member of a single-primed family, satisfying assumptions I through $\mathrm{V}$, is a union of primary members of the family. For this special case the result is achieved without making assumptions (a) and (b) of $[2, \S 7]$.

\title{
BIBLIOGRAPHY
}

1. W. Krull, Idealtheorie, Ergebnisse der Mathematik, vol. 4, Part III, Berlin, 1935.

2. R. E. MacKenzie, Commutative semigroups, Duke Math. J. vol. 21 (1954) pp. $471-477$.

3. B. L. van der Waerden, Modern algebra, vol. 2, 2d ed., New York, 1950.

Texas Technological College

\section{A LIMIT THEOREM FOR PROCESSES WITH STATIONARY INDEPENDENT INCREMENTS}

FRANK KOZIN

I. Introduction. Let $[X(t) ; 0 \leqq t \leqq 1]$ be the Wiener process. That is, a Gaussian process of real valued variables with $E[X(t)]=0$ and $E[X(s) X(t)]=\min (s, t)$. Levy [1] showed that with probability one

$$
\lim _{N \rightarrow \infty} \sum_{k=1}^{N}\left[X\left(t_{k}\right)-X\left(t_{k-1}\right)\right]^{2}=1 \quad \text { for } 0=t_{0}<t_{1}<\cdots<t_{N}=1 .
$$

Cameron and Martin [2] showed this independently for $t_{k}=k / 2^{N}$. Baxter [3] recently extended these results by the following theorem:

THEOREM. If $[X(t) ; 0 \leqq t \leqq 1]$ is a Gaussian process satisfying $E[X(t)]=m(t)$ and $E[X(s) X(t)]-m(s) m(t)=r(s, t), r(s, t)$ is continuous in $0 \leqq s, t \leqq 1$ and has uniformly bounded second derivatives for $s \neq t$.

Let

$$
\begin{aligned}
& D^{+}(t)=\lim _{s \rightarrow t^{+}} \frac{r(t, t)-r(s, t)}{t-s}, \\
& D^{-}(t)=\lim _{s \rightarrow t^{-}} \frac{r(t, t)-r(s, t)}{t-s},
\end{aligned}
$$

and

$$
f(t)=D^{-}(t)-D^{+}(t) .
$$

Then with probability one

Received by the editors November 2, 1956. 


$$
\lim _{N \rightarrow \infty} \sum_{k=1}^{2 N}\left[X\left(\frac{k}{2^{N}}\right)-X\left(\frac{k-1}{2^{N}}\right)\right]^{2}=\int_{0}^{1} f(t) d t .
$$

Using Baxter's procedure we obtain a similar but more general result since we place little restriction on the values $t_{k}$ at which the increments are taken.

Processes with stationary independent increments are characterized by their characteristic functions which are of the form $E[\exp (i u X(t))]=\exp (t h(u))$, where $h(0)=0$.

Our main result is the following theorem:

TheOREM I.: Let $[X(t) ; 0 \leqq t \leqq 1]$ be a process with stationary independent increments having characteristic function $\exp (\operatorname{th}(u))$. Let $E\left[X(t)^{4}\right]$ exist and $\left.D^{4} h(u)\right|_{u=0}=0$. If $0=t_{0}<t_{1}<\cdots<t_{N_{n}}=1$ and $\lim _{n \rightarrow \infty} \Delta t_{\max }=o\left(n^{-2}\right)$, where $\Delta t_{\max }=\max \left[t_{k}-t_{k-1}\right], k=1, \cdots, N_{n}$, then with probability one

$$
\lim _{n \rightarrow \infty} \sum_{k=1}^{N_{n}}\left[X\left(t_{k}\right)-X\left(t_{k-1}\right)\right]^{2}=-D^{2} h(0) .
$$

II. Proof of theorem. We first assume that the mean is equal to zero. That is, $E[X(t)]=0$. Since the fourth moment exists, then

$$
\left.D^{i} \exp (\operatorname{th}(u))\right|_{u=0}=i^{i} E\left[X(t)^{i}\right], \quad j=1,2,3,4 .
$$

Now for $k=1, \cdots, N_{n}$ we let

$$
\begin{aligned}
\Delta X_{k} & =X\left(t_{k}\right)-X\left(t_{k-1}\right), \\
\Delta t_{k} & =t_{k}-t_{k-1} .
\end{aligned}
$$

Then we have, using (2),

$$
E\left[\sum_{k=1}^{N_{n}} \Delta X_{k}^{2}\right]=\sum_{k=1}^{N_{n}} E\left[\Delta X_{k}^{2}\right]=\sum_{k=1}^{N_{n}}-D^{2} h(0) \Delta t_{k}=-D^{2} h(0) .
$$

Notice that this is independent of $N_{n}$. Thus in the limiting case we obtain the same value.

Using (2) and the hypothesis, we obtain

$$
\begin{aligned}
E\left[\left[\sum_{k=1}^{N_{n}} \Delta X_{k}^{2}\right]^{2}\right] & =\left(D^{2} h(0)\right)^{2}\left[3 \sum_{k=1}^{N_{n}} \Delta t_{k}^{2}+2 \sum_{k \neq j=1}^{N_{n}} \Delta t_{k} \Delta t_{j}\right] \\
& =\left(D^{2} h(0)\right)^{2}\left[2 \sum_{k=1}^{N_{n}} \Delta t_{k}^{2}+\left[\sum_{k=1}^{N_{n}} \Delta t_{k}\right]^{2}\right] \\
& =\left(D^{2} h(0)\right)^{2}\left[2 \sum_{k=1}^{N_{n}} \Delta t_{k}^{2}+1\right] .
\end{aligned}
$$


Thus applying the Tchebychev inequality, we obtain

$$
\begin{aligned}
P\left\{\left|\sum_{k=1}^{N_{n}} \Delta X_{k}^{2}-E\left[\sum_{k=1}^{N_{n}} \Delta X_{k}^{2}\right]\right| \geqq n\left(\Delta t_{\max }\right)^{1 / 2}\right\} \\
\leqq \frac{2\left(D^{2} h(0)\right)^{2} \sum_{k=1}^{N_{n}} \Delta t_{k}^{2}}{n^{2} \Delta t_{\max }} \leqq \frac{2\left(D^{2} h(0)\right)^{2} \sum_{k=1}^{N_{n}} \Delta t_{k}}{n^{2}}=\frac{2\left(D^{2} h(0)\right)^{2}}{n^{2}},
\end{aligned}
$$

which is a term of a convergent series. Hence, by the Borel-Cantelli lemma and the hypothesis concerning $\Delta t_{\max }$, the expression within the absolute value signs has the limit zero with probability one as $n$ becomes infinite. Upon writing

$$
\sum_{k=1}^{N_{n}} \Delta X_{k}^{2}=E\left[\sum_{k=1}^{N_{n}} \Delta X_{k}^{2}\right]+\left[\sum_{k=1}^{N_{n}} \Delta X_{k}^{2}-E\left[\sum_{k=1}^{N_{n}} \Delta X^{2}\right]\right],
$$

and using the result of (5), the theorem is proved in the case that the mean is zero.

Now in the case that the mean is not equal to zero we form a new process $[\bar{X}(t) ; 0 \leqq t \leqq 1]$, where $\bar{X}(t)=X(t)-m(t)$ and $m(t)$ is the mean function. From (2), $m(t)=-i t D h(0)$. From the proof above

$$
\lim _{n \rightarrow \infty} \sum_{k=1}^{N_{n}} \Delta \bar{X}_{k}^{2}=-D^{2} h(0)
$$

with probability one. Upon setting $\Delta m_{k}=m\left(t_{k}\right)-m\left(t_{k-1}\right)$, we have

$$
\sum_{k=1}^{N_{n}} \Delta X_{k}^{2}=\sum_{k=1}^{N_{n}} \Delta \bar{X}_{k}^{2}+2 \sum_{k=1}^{N_{n}} \Delta m_{k} \Delta \bar{X}_{k}+\sum_{k=1}^{N_{n}} \Delta m_{k}^{2}
$$

From (2) we have

$$
\sum_{k=1}^{N_{n}} \Delta m_{k}^{2}=\sum_{k=1}^{N_{n}}-(D h(0))^{2} \Delta t_{k}^{2} \leqq-(D h(0))^{2} \Delta t_{\max } .
$$

By the Schwarz inequality we have

$$
\left[\sum_{k=1}^{N_{n}} \Delta m_{k} \Delta \bar{X}_{k}\right]^{2} \leqq \sum_{k=1}^{N_{n}} \Delta m_{k}^{2} \sum_{k=1}^{N_{n}} \Delta \bar{X}_{k}^{2}
$$

where the second term on the right is bounded with probability one. By (7) the first term on the right of (8) approaches zero as $n$ becomes infinite. Thus

$$
\lim _{n \rightarrow \infty} \sum_{k=1}^{N_{n}} \Delta X_{k}^{2}=\lim _{n \rightarrow \infty} \sum_{k=1}^{N_{n}} \Delta \bar{X}_{k}^{2}=-D^{2} h(0) .
$$


Hence Theorem I is proved.

III. Conclusion. Clearly Baxter's theorem and the theorem above overlap for Gaussian processes with stationary independent increments, that is, Wiener processes. We also mention the fact that Baxter's theorem can be proven for more general $t_{k}$ using the same method as in the theorem above.

In closing we mention one very simple but interesting consequence of Theorem I. If $P(x, t)$ is the distribution function associated with the process, then if $t_{1}+\cdots+t_{n}=1$ and $S_{n}(R)$ is an $n$-dimensional sphere of radius $R$,

$$
\begin{aligned}
f(R) & =\lim _{n \rightarrow \infty} \int \cdots \int_{S n(R)} D P\left(x_{1}, t_{1}\right) \cdots D P\left(x_{n}, t_{n}\right) \\
& = \begin{cases}1, & R \geqq\left(-D^{2} h(0)\right)^{1 / 2} \\
0, & R<\left(-D^{2} h(0)\right)^{1 / 2}\end{cases}
\end{aligned}
$$

\section{BIBLIOGRAPHY} 550.

1. P. Lévy, Le mouvement brownien plan, Amer. J. Math. vol. 62 (1940) pp. 487-

2. R. H. Cameron and W. T. Martin, The behavior of measure and measurability under a change of scale in Wiener space, Bull. Amer. Math. Soc. vol. 53 (1947) pp. 130137.

3. G. Baxter, A strong limit theorem for Gaussian processes, Proc. Amer. Math. Soc. vol. 7 (1956) pp. 522-527.

Purdue University 